\title{
Multifocal optical-resolution photoacoustic microscopy in reflection mode
}

Guo Li, Konstantin I. Maslov, Lihong V. Wang

Guo Li, Konstantin I. Maslov, Lihong V. Wang, "Multifocal optical-resolution photoacoustic microscopy in reflection mode

," Proc. SPIE 8581, Photons Plus Ultrasound: Imaging and Sensing 2013, 858126 (4 March 2013); doi: 10.1117/12.2005673

SPIE. Event: SPIE BiOS, 2013, San Francisco, California, United States 


\title{
Multifocal optical-resolution photoacoustic microscopy in reflection mode

\author{
Guo Li, Konstantin I. Maslov, and Lihong V. Wang*
}

\author{
Optical Imaging Laboratory, Department of Biomedical Engineering, Washington University in St. \\ Louis, One Brookings Drive, St. Louis, Missouri 63130 \\ *Corresponding author: 1hwang@biomed.wustl.edu
}

\begin{abstract}
Compared with single-focus optical-resolution photoacoustic microscopy (OR-PAM), multifocal OR-PAM utilizes both multifocal optical illumination and an ultrasonic array transducer, significantly increasing the imaging speed. Here we present a reflection-mode multifocal OR-PAM system based on a microlens array that provides multiple foci and an ultrasonic array transducer that receives the excited photoacoustic waves from all foci simultaneously. By using a customized microprism to reflect the incident laser beam to the microlens array, we align the multiple optical foci confocally with the focal zone of the ultrasonic array transducer. Experiments show our reflection-mode multifocal ORPAM system is capable of imaging microvessels in vivo, and it can image a $9 \mathrm{~mm} \times 5 \mathrm{~mm} \times 2.5 \mathrm{~mm}$ volume at $16 \mu \mathrm{m}$ lateral resolution in $\sim 4 \mathrm{~min}$, limited by the signal multiplexing ratio and laser pulse repetition rate.
\end{abstract}

Keywords: photoacoustic microscopy; high-frequency ultrasonic array; multiple foci; reflection mode.

\section{INTRODUCTION}

Photoacoustic microscopy (PAM) is a mode of photoacoustic tomography which offers high sensitivity to optical contrast in biological tissues. ${ }^{1}$ Optical-resolution PAM (OR-PAM) is implemented by focusing the illumination through an objective lens; thus, OR-PAM can achieve optical diffraction limited lateral resolution with maximum imaging depths up to one transport mean-free path $(\sim 1.2 \mathrm{~mm}$ in tissue $){ }^{2,3}$ OR-PAM can provide structural imaging and metabolic imaging. ${ }^{4,5}$ Various kinds of OR-PAM have also been explored to meet specific requirements, such as high speed, ${ }^{6}$ high resolution, ${ }^{7,8}$ deep imaging depth, ${ }^{3}$ and handheld operation ${ }^{9}$.

For OR-PAM, high imaging speed is always desirable because it enables us to observe dynamic biological processes while mitigating motion artifacts. For most OR-PAM systems based on both a single optical focus and a single-element ultrasonic transducer in confocal configuration, mechanical raster scanning along two lateral directions provides limited volumetric imaging speeds. Although optical scanning (scanning using galvo mirrors) can improve the speed greatly, it is usually used with an unfocused or a single-axis focused ultrasonic transducer, which results in a limited field of view or compromised signal-to-noise ratio. Moreover, the volumetric imaging speed for OR-PAM with either mechanical or optical scanning is eventually limited by the laser pulse repetition rate (PRR). Thus to achieve high imaging speed, lasers with high PRR ( $\sim 100 \mathrm{kHz}$, or even faster) are employed. ${ }^{6,9}$ Currently, lasers with such high PRR usually lack the wavelength tunability essential for imaging of some physiological functions, such as the oxygen saturation of hemoglobin $\left(\mathrm{sO}_{2}\right)$. Moreover, high PRR translates to high average optical power, which may produce excessive tissue heating. Our lab previously reported a multifocal OR-PAM (MFOR-PAM) with $\sim 20$ times faster imaging speed (without considering the multiplexing effect) than a single-focus OR-PAM; ${ }^{10}$ thus relieving the demand for a high laser PRR. Besides the imaging speed improvement, the $\mathrm{sO}_{2}$ measurement capability makes MFOR-PAM potentially promising in clinical and preclinical applications. However, the original MFOR-PAM works in transmission mode, only suited for imaging thin biological samples. Therefore, it is necessary to develop reflection-mode MFOR-PAM.

Photons Plus Ultrasound: Imaging and Sensing 2013, edited by Alexander A. Oraevsky, Lihong V. Wang, Proc. of SPIE Vol. 8581, 858126 - (C) 2013 SPIE · CCC code: 1605-7422/13/\$18 - doi: 10.1117/12.2005673 
Although there are numerous ways to realize reflection-mode single-focus OR-PAM, ${ }^{8}$ the characteristics of the existing ultrasonic array transducer and the microlens array make it difficult to realize reflection-mode MFOR-PAM. First, the existing curved cylindrical acoustic lens and antireflection coating of the transducer array preclude directly attaching a light-reflecting combiner ${ }^{11}$ (Fig. 1). Adding a detached combiner would generate at least two more ultrasonic reflection interfaces with the acoustic coupling medium, which cause considerable photoacoustic signal loss. For example, two interfaces between BK7 glass and water would cause $\sim 90 \%$ acoustic energy loss (10 dB transmission). Second, the elevational (transverse) size of the ultrasonic array transducer is larger than the focal length of a closely packed lenslet array. Hence, it is impossible to insert a sound-reflecting combiner between the lenslet array and tissue surface.

\section{EXPERIMENTAL SETUP}

We implemented the reflection-mode MFOR-PAM by placing a microlens array directly below the ultrasonic array transducer, with both immersed in water, as shown in Fig. 1. To get one-dimensional multifocal optical illumination, the microlens array (250 $\mu \mathrm{m}$ pitch and $1.2 \mathrm{~mm}$ focal length in air, 11-1640-123-000, SUSS MicroOptics, Switzerland) was precisely cut by Biomedical-Optics LLC to $0.5 \mathrm{~mm}$ wide and $10 \mathrm{~mm}$ long. At a laser wavelength of $570 \mathrm{~nm}$, Zemax (Radiant Zemax LLC) simulation shows that the water-immersed microlens can produce a diffraction-limited focus with a radius of $\sim 7 \mu \mathrm{m}$ at the waist and an effective focal length of $3.4 \mathrm{~mm}$. The centers of the microlens array and the ultrasonic array transducer were aligned in both the elevational (x axis in Fig. 1) and lateral (y axis in Fig. 1) directions. The microlens array was carefully adjusted so that the optical foci of the microlens array and the cylindrical focus of ultrasonic array transducer overlap. Such a confocal configuration optimizes the detection sensitivity. To reflect the laser beam, which is incident from the elevational side, a customized microprism $(0.5 \mathrm{~mm}$ wide $\times 0.5 \mathrm{~mm}$ high $\times 7 \mathrm{~mm}$ long, Biomedical-Optics LLC) was used. The microprism and the microlens array were aligned on their centers and glued together with optically transparent adhesive.

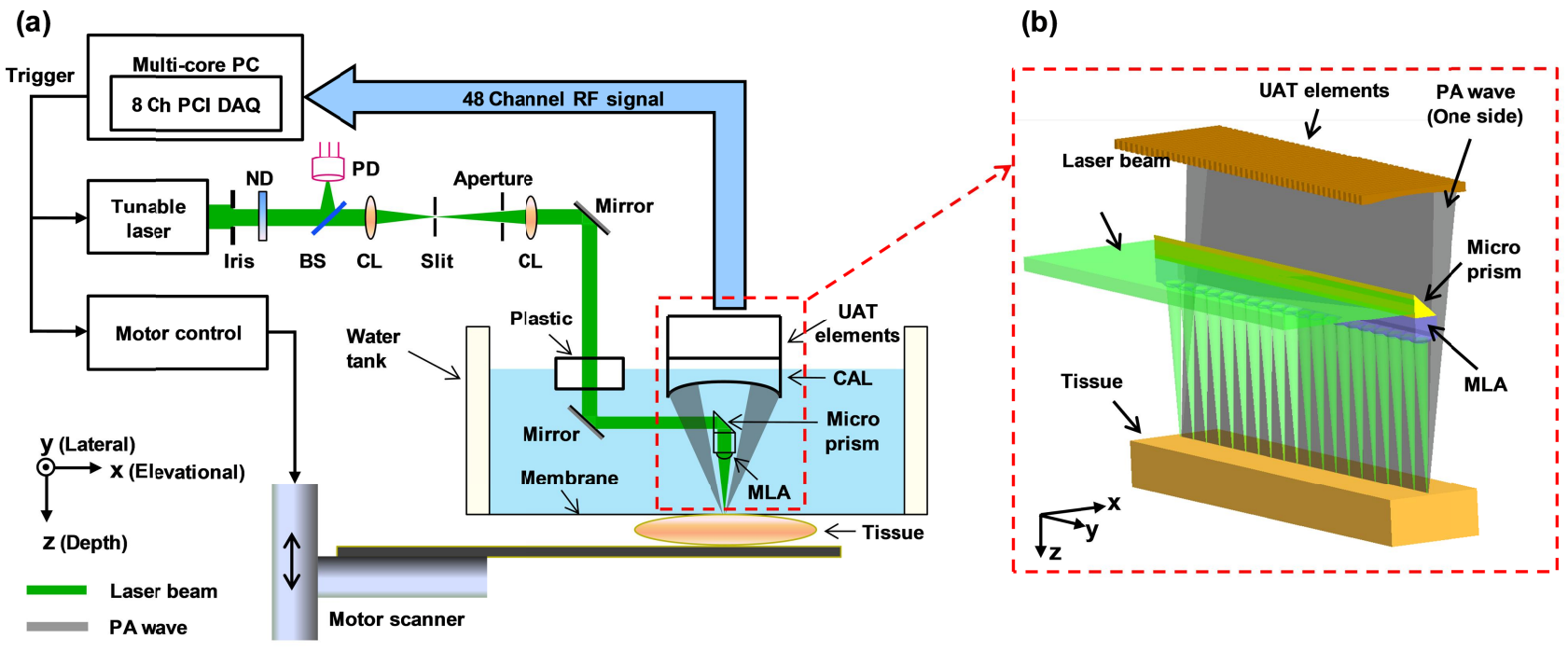

Fig. 1. Configuration of the reflection-mode MFOR-PAM system. (a) Schematic of the whole system. BS, beam sampler; CL, convex cylindrical lens; CAL, cylindrical acoustic lens; MLA, microlens array; ND, neutral density filter; PD, photodiode; UAT, ultrasonic array transducer. (b) Three-dimensional view of the components in the dashed box of (a). To show the configuration more clearly, only one side of the photoacoustic wave is presented.

A tunable dye laser (Cobra, Sirah, Germany) pumped by a Q-switched Nd:YLF laser (INNOSLAB, EdgeWave, Germany) with PRR up to $1.35 \mathrm{kHz}$ provided photoacoustic excitation. The laser beam was first focused by a convex cylindrical lens. After passing through the slit, the beam was slightly focused by an objective cylindrical lens onto the customized microprism. In this study, due to the limited detection range of the ultrasonic array transducer, only 20 microlenses were used to provide 20 focused optical illumination spots along the lateral axis. Optically transparent plastic (Poly(methyl methacrylate), PMMA) was used to couple the light from air into water and remove possible water ripples during scanning. The linear ultrasonic array transducer simultaneously detected photoacoustic waves from all 
illumination beams. The ultrasonic array transducer consisted of 48 elements with $100 \mu \mathrm{m}$ pitch, and each element (82 $\mu \mathrm{m} \times 2 \mathrm{~mm}$ ) had a central frequency of $30 \mathrm{MHz}$ and a $70 \%$ receiving-only bandwidth. The amplified 48 channel signals were then multiplexed to an 8-channel data acquisition card (Octopus 8389, Gage Applied Technologies, USA). More technical details about this ultrasound array system can be found in our previous publications. ${ }^{10,12}$ To acquire volumetric photoacoustic images, two scanning motors were implemented to scan the tissue as well as the water tank along the elevational and lateral directions. The scanning motors were also controlled by triggers from the data acquisition card. A single-focus OR-PAM system must scan the front-to-end length of the 20 microlenses $(20 \times 250 \mu \mathrm{m})$ in the lateral direction. However, because of its simultaneous illumination, the MFOR-PAM system needs to scan only one pitch (250 $\mu \mathrm{m}$ ) of the microlens along the lateral direction. Thus, in theory, the MFOR-PAM system has twentyfold faster scanning capability than single-focus OR-PAM at the same laser PRR.

In this reflection-mode MFOR-PAM, the photoacoustic wave was partially reflected by the microlens array when propagating to the transducer. To estimate the fractional photoacoustic wave loss, we used the following parameters: $f_{1}$ and $w_{1}$, focal length and width of the microlens array immersed in water; $f_{2}$, focal length of the cylindrical acoustic lens; $w_{2}$, width of the transducer element; $w^{\prime}$, portion of the width that cannot receive photoacoustic waves. The fractional photoacoustic wave loss is $w^{\prime} / w_{2}=w_{1} f_{2} /\left(w_{2} f_{1}\right)$. Here we neglect the acoustic diffraction effect because the acoustic wavelength is much smaller than the width of the microlens array. With $f_{1}=3.4 \mathrm{~mm}, w_{1}=0.5 \mathrm{~mm}, f_{2}=8.2 \mathrm{~mm}$, and $w_{2}=$ $2.0 \mathrm{~mm}$, the fraction of acoustic energy reaching the transducer array, was $1-w / w_{2}$, equal to $\sim 40 \%$. Also, this design allows us to extend the microscope's working distance to the whole optical focal length, which is convenient in biomedical imaging applications.

\section{RESULTS}

In MFOR-PAM, the lateral resolution and elevational resolution are the same because of the symmetric property of the optical focus. ${ }^{10}$ Therefore, hereafter we use "lateral resolution" to denote both the lateral resolution and elevational resolution. To quantify the lateral resolution of the reflection-mode MFOR-PAM, we imaged two crossed 6- $\mu$ m-diameter carbon fibers, using a scanning step size of $2 \mu \mathrm{m}$. By using the Gaussian fitting method to the PA signal amplitude distribution along the direction which is perpendicular to the carbon fiber, we got the full width at half maximum (FWHM) value of $16 \mu \mathrm{m}$, which represents the lateral resolution of the system. Due to the water immersion, this value is lower than the $10 \mu \mathrm{m}$ lateral resolution of the transmission mode MFOR-PAM. The axial resolution, which is determined by the receiving ultrasonic bandwidth of the transducer, is $\sim 25 \mu \mathrm{m}$, as reported in previous publications. ${ }^{10,}{ }^{12}$ The penetration depth of the reflection-mode MFOR-PAM was quantified to be $0.7 \mathrm{~mm}$ by imaging a $250 \mu \mathrm{m}$-diameter black needle obliquely inserted into the leg of a nude mouse (Hsd, Athymic Nude-Foxnlnu, Harlan Co.). Compared to the maximum imaging depths of other OR-PAM systems (up to $1.2 \mathrm{~mm}$ ), ${ }^{2,6,11}$ this depth is shallower because the microlens array reflects $\sim 60 \%$ of the PA wave. However, this maximum imaging depth is still sufficient for observations of many shallow structures.

Fig. 2 shows an in vivo maximum amplitude projection (MAP) image of a mouse ear microvasculature acquired by the reflection-mode MFOR-PAM system. The MAP image was processed by the $2 \times 2$ Gaussian low pass filter. The laser wavelength was set to the isosbestic wavelength of $570 \mathrm{~nm}$, where hemoglobin has high absorption. Microvessels were clearly imaged in the MAP image. The imaging time to acquire the data set of Fig. $2(900 \times 500 \times 200$ voxels $)$ was $\sim 4$ min. The incident laser energy on each microlens was limited to $\sim 62 \mathrm{~nJ} / \mathrm{pulse}$, and the laser fluence on the ear surface was estimated to be $16 \mathrm{~mJ} / \mathrm{cm}^{2}$, lower than the ANSI limit $20 \mathrm{~mJ} / \mathrm{cm}^{2}$.

With the flexibility of reflection mode, the in vivo photoacoustic image of a nude mouse brain was also acquired. Fig. 3 shows the MAP image of a living adult mouse brain. The MAP image was processed by the $2 \times 2$ Gaussian low pass filter. The skin of the mouse brain was removed, but the skull was kept intact. From Fig. 3, the microvessels can be clearly observed, even though the intact skull attenuated both the incident light and the PA wave. 


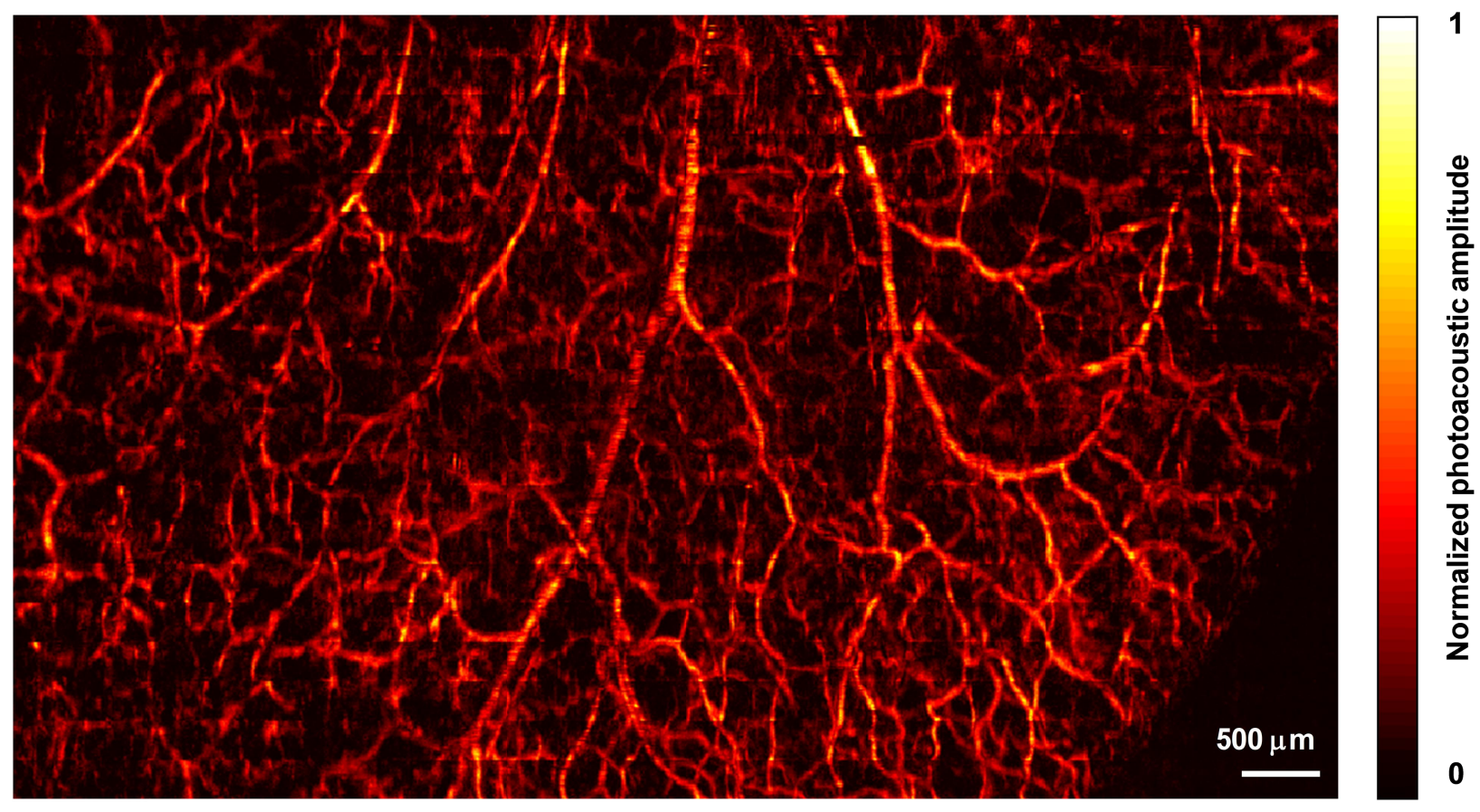

Fig. 2. In vivo photoacoustic MAP image of a nude mouse ear

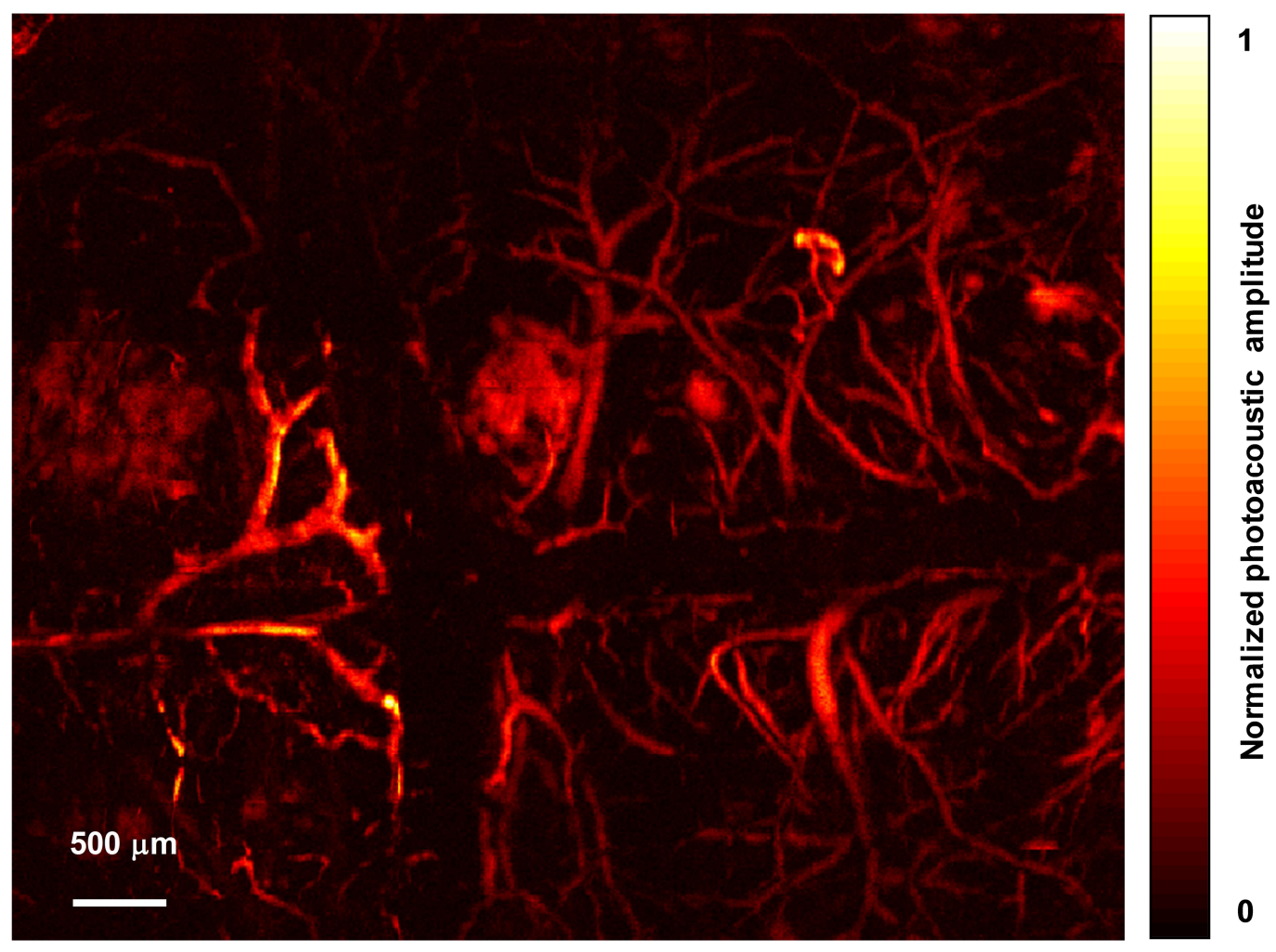

Fig. 3 In vivo trans-cranial photoacoustic MAP images of a nude mouse brain with the skin removed

Proc. of SPIE Vol. $8581858126-4$ 


\section{CONCLUSION AND FUTURE WORK}

We have developed a reflection-mode multifocal optical-resolution photoacoustic microscopy (MFOR-PAM) system using a microlens array and a linear ultrasonic array transducer. The reflection-mode MFOR-PAM was capable of acquiring volumetric photoacoustic images in vivo, with a lateral FWHM resolution of $16 \mu \mathrm{m}$, axial resolution of $\sim 25$ $\mu \mathrm{m}$, and in vivo penetration depth up to $0.7 \mathrm{~mm}$. Due to the combination of multiple optical foci and the ultrasonic array transducer, the lateral scanning range was reduced to only one pitch of the microlens array; thus, the imaging speed was much faster than single-focus OR-PAM with mechanical scanning. Potentially the imaging speed can be further improved by eliminating the 6:1 multiplexer, and using a higher PRR laser. Since the mechanical scanning motors in this experiment can achieve a speed of $20 \mathrm{~mm} / \mathrm{s}$ (bi-directional scanning with $10 \mathrm{~mm}$ scanning length in each direction), without multiplexing it is possible to achieve an imaging speed of $(20 \mathrm{~mm} / \mathrm{s}) /(10 \mu \mathrm{m} / \mathrm{voxel}) \times(200$ voxels/A-line $) \times 20$ lenslets $=8 \times 10^{6}$ voxels per second (voxel size: $10 \mu \mathrm{m} \times 10 \mu \mathrm{m} \times 25 \mu \mathrm{m}$ ). Optical scanning within one pitch in the lateral direction can further improve the imaging speed by several times. A more densely packed microlens array with a smaller pitch might improve the imaging speed, but this usually means a shorter working distance if we want to maintain the NA or lateral resolution. Other multifocal designs might be developed to provide both a high NA and long working distance.

\section{ACKNOWLEDGMENTS}

This work was sponsored in part by National Institutes of Health (NIH) grants R01 EB000712, R01 EB008085, R01 CA134539, U54 CA136398, R01 CA157277, R01 CA159959, and DP1 EB016986 (NIH Director's Pioneer Award). L. V. Wang has a financial interest in Microphotoacoustics, Inc., and Endra, Inc., which, however, did not support this work. K. I. Maslov has a financial interest in Microphotoacoustics, Inc., which did not support this work. The authors thank Liang Song, Lidai Wang, Junjie Yao, and Jun Xia for helpful discussions and experimental assistance.

\section{REFERENCES}

[1] Wang, L. V., "Multiscale photoacoustic microscopy and computed tomography," Nat Photon 3(9), 503-509 (2009)

[2] Hu, S., Maslov, K. and Wang, L. V., "Second-generation optical-resolution photoacoustic microscopy with improved sensitivity and speed," Opt. Lett. 36(7), 1134-1136 (2011)

[3] Yao, J., Maslov, K. I., Puckett, E. R., Rowland, K. J., Warner, B. W. and Wang, L. V., "Double-illumination photoacoustic microscopy," Opt. Lett. 37(4), 659-661 (2012)

[4] Hajireza, P., Shi, W.and Zemp, R. J., "Label-free in vivo fiber-based optical-resolution photoacoustic microscopy," Opt. Lett. 36(20), 4107-4109 (2011)

[5] Krumholz, A., Wang, L., Yao, J.and Wang, L. V., "Functional photoacoustic microscopy of diabetic vasculature," J. Biomed. Opt. 17(6), 060502-060501 (2012)

[6] Yao, J., Huang, C.-H., Wang, L., Yang, J.-M., Gao, L., Maslov, K. I., Zou, J.and Wang, L. V., "Wide-field fastscanning photoacoustic microscopy based on a water-immersible MEMS scanning mirror," J. Biomed. Opt. 17(8), 080505-080501 (2012)

[7] Zhang, C., Maslov, K. and Wang, L. V., "Subwavelength-resolution label-free photoacoustic microscopy of optical absorption in vivo," Opt. Lett. 35(19), 3195-3197 (2010)

[8] Zhang, C., Maslov, K., Hu, S., Chen, R., Zhou, Q., Shung, K. K.and Wang, L. V., "Reflection-mode submicronresolution in vivo photoacoustic microscopy," J. Biomed. Opt. 17(2), 020501-020504 (2012) 
[9] Hajireza, P., Shi, W.and Zemp, R. J., "Real-time handheld optical-resolution photoacoustic microscopy," Opt. Express 19(21), 20097-20102 (2011)

[10] Song, L., Maslov, K. and Wang, L. V., "Multifocal optical-resolution photoacoustic microscopy in vivo," Opt. Lett. 36(7), 1236-1238 (2011)

[11] Wang, L., Maslov, K., Yao, J., Rao, B. and Wang, L. V., "Fast voice-coil scanning optical-resolution photoacoustic microscopy," Opt. Lett. 36(2), 139-141 (2011)

[12] Zemp, R. J., Song, L., Bitton, R., Shung, K. K. and Wang, L. V., "Realtime photoacoustic microscopy in vivo with a 30-MHz ultrasound array transducer," Opt. Express 16(11), 7915-7928 (2008) 